\section{Steroide sparen durch lang wirksame $\beta$-Mimetika?}

ann man bei Asthmatikern durch langwirksame Betamimetika inhalative Steroide einsparen? Antworten geben jetzt zwei in der Zeitschrift JAMA publizierte Studien und sie lauten nein bzw. jein:

Wenn Asthmapatienten ihre Symptomatik mit einem inhalativen Steroid gut unter Kontrolle haben, macht es keinen Sinn, auf Salmeterol zu wechseln. Die Folge sind mehr Therapieversager und Exazerbationen.

\title{
Aus Schreihälsen werden Atopiker
}

$\mathrm{H}$

äufiges Quengeln und Schreien bei Kleinkindern sind Prädiktoren für die spätere Ausbildung einer Atopie - ein zugegeben überraschender Zusammenhang, den finnische Forscher jetzt mit einer prospektiven Untersuchung an 116 Kindern mit familiärem Atopie-Risiko nachweisen wollten. Während der siebten und der zwölften Lebenswoche mussten die Eltern penibel das Verhalten ihres Säuglings protokollieren. Zwei Jahre später waren insgesamt 44 der Kinder durch eine atopische Symptomatik
Wenn Asthmapatienten hingegen ihre Symptomatik mit einem inhalativen Steroid nicht völlig unter Kontrolle bringen, macht es sehr wohl Sinn, Salmeterol zusätzlich zu verordnen. Bei gemeinsamer Gabe kann sogar die Steroiddosis halbiert werden, ohne negative Folgen. red

Lazarus SC et al. JAMA 2001; 285:

2583-93

Lemanske RF et al. JAMA 2001; 285 :

2594-603 aufgefallen und zwar signifikant häufiger in der Tat eben jene Kinder, die sich in den ersten Lebenswochen besonders lautstark bemerkbar gemacht hatten. Auch eine plausible Erklärung für ihre Beobachtung bleiben die Skandinavier nicht schuldig: Hauptgrund für das kindliche Unwohlsein seien ja Darmkoliken, und die Darmflora habe schließlich etwas mit dem Immunsystem zu tun ...

es

Kalliomaki M et al. Arch Dis Child 2001; 84:349-50

\section{Infektprävention mit Probiotika}

$M$ anche Bio-Mutter mag es freuen, und die Lebensmittelindustrie ebenfalls: Probiotische Milchprodukte üben bei Kindern eine gewisse Schutzfunktion vor Infektionen aus. Wie eine im „British Medical Journal“ publizierte Doppelblindstudie zeigte, reduzierten die Lactobazillen-Produkte die Inzidenz von Atemwegsinfektionen und den Antibiotikaverbrauch um knapp 20\%. Ein BMJ-Editorial stellt dazu fest, dass sich die Hinweise mehren, dass Probiotika Kindern mit erhöhter Gefährdung für Atemwegs- und Durchfallerkrankungen nützen. (siehe auch S. 244)

Hatakka K et al. BMJ 2001; 322: 1327

\section{Haben dralle Dirndl enge Bronchien?}

E ast 10.000 Mädchen und Jungen aus ländlichen Gegenden Bayerns füllten im Auftrag der Münchner Ludwig-Maximilians-Universität Fragebögen aus. Ziel der Untersuchung war es, einem möglichen Zusammenhang zwischen Übergewicht und asthmatischen Erkrankungen auf die Spur zu kommen. Die Suche war, was die Knaben betrifft, ergebnislos. Bei den fünf- bis sechsjährigen Mädchen ergab sich allerdings eine deutliche Korrelation: Lag die Asthma-Prävalenz bei den normalgewichtigen Mädchen bei 3,5\%, war sie bei den übergewichtigen auf $5,8 \%$ und bei den adipösen auf $10,3 \%$ erhöht. Als eine mögliche Ursache für diese Beobachtung mutmaßen die Forscher, dass die geschlechtsspezifische Fettverteilung bei Mädchen eine relative Verhttp://www.ageh.de abrufen oder direkt bei der AGEH Ripuarenstraße 8, D-50679 Köln, Tel. 02 21/88 96-0, Fax 02 21/88 96-100, anfordern. red engung der Atemwege zur Folge hat. es

von Kries $\mathbf{R}$ et al. Allergy 2001; 56: 318-22 